\title{
Caracterização de frutos de Dialium Guianense cultivados na Região Norte do Brasil
}

\author{
Characterization of Dialium Guianense fruits grown in the Northern Region of Brazil \\ Caracterización de frutos de Dialium Guianense cultivados em la Región Norte de Brasil
}

Recebido: 01/12/2021 | Revisado: 09/12/2021 | Aceito: 14/12/2021 | Publicado: 22/12/2021

Vitória Cláudia Oliveira Machado
ORCID: https://orcid.org/0000-0001-5533-4022
E-mail: vicomachado2002@gmail.com
Sávio Ferreira de Freitas
Instituto Federal de Educação, Ciência e Tecnologia de Roraima, Brasil
ORCID: https://orcid.org/0000-0001-6423-121X
E-mail: saviohtj@gmail.com
Wanderson Morais de Sousa
Instituto Federal de Educação, Ciência e Tecnologia de Roraima, Brasil
ORCID: https://orcid.org/0000-0003-2221-7991
E-mail: wandersonmoraisdesousa@gmail.com
Brainer William Cruz dos Santos
ORCID: https://orcid.org/0000-0003-0421-4690
Instituto Federal de Educaçân,
E-mail: brainer.cruz@ifrr.edu.br
Tassiane Dos Santos Ferrão
Instituto Federal de Educação, Ciência e Tecnologia de Roraima, Brasil
ORCID: https://orcid.org/0000-0003-0824-7094
E-mail: tassiane.ferrao@ifrr.edu.br

\section{Resumo}

Dialium Guianense, popularmente conhecida como Pororoca ou Jutaí, é uma fruta exótica cultivada em áreas da região amazônica. Este trabalho teve como objetivo realizar a caracterização biométrica e físico-química de frutos de Dialium Guianense. Para a avaliação dos parâmetros biométricos, vinte frutos inteiros foram pesados e medidos (comprimento e largura) individualmente. A análise de cor foi realizada em colorímetro Delta. A composição centesimal e valor calórico da polpa da fruta foram determinados por meio das análises de umidade, cinzas, lipídios, proteína bruta, fibra bruta e carboidratos. O resultado dos parâmetros biométricos e análise de cor demonstraram que a pororoca se trata de um fruto pequeno $(21,09 \times 12,18 \mathrm{~mm}$ e $0,82 \mathrm{~g})$ com casca marrom e polpa de coloração laranja. Quanto aos resultados da composição química, a polpa de pororoca apresentou $28,26 \%$ de umidade; $71,74 \%$ de matéria seca; $3,37 \%$ de cinzas; $0,45 \%$ de lipídeos; $2,77 \%$ de proteína bruta; $5,07 \%$ de fibra bruta; e $60,07 \%$ de carboidrato. A estimativa de valor calórico revelou que a fruta possui $255 \mathrm{kcal} \mathrm{g}^{-1}$. Conclui-se que a caracterização físico-química se torna importante por fornecer dados que podem ser usados pelas indústrias para a obtenção de novos produtos alimentícios.

Palavras-chave: Análise biométrica; Cor; Composição química; Peso; Pororoca.

\begin{abstract}
Dialium Guianense, popularly known as Pororoca or Jutaí, is an exotic fruit cultivated in areas of the Amazon region. This work aimed to carry out the biometric and physicochemical characterization of Dialium Guyanense fruits. For the evaluation of biometric parameters, twenty whole fruits were weighed and measured (length and width) individually. Color analysis was performed using a Delta colorimeter. The proximate composition and caloric value of the fruit pulp were determined by analyzing moisture, ash, lipids, crude protein, crude fiber and carbohydrates. The result of the biometric parameters and color analysis showed that the pororoca is a small fruit $(21.09 \times 12.18 \mathrm{~mm}$ and $0.82 \mathrm{~g}$ ) with a brown skin and orange colored pulp. As for the results of the chemical composition, the pororoca pulp presented $28.26 \%$ moisture; $71.74 \%$ dry matter; $3.37 \%$ ash; $0.45 \%$ lipids; $2.77 \%$ crude protein; $5.07 \%$ crude fiber; and $60.07 \%$ carbohydrate. The caloric value estimate revealed that the fruit has $255 \mathrm{kcal} \mathrm{g}-1$. It is concluded that the physicochemical characterization becomes important for providing data that can be used by industries to obtain new food products.
\end{abstract}

Keywords: Biometric analysis; Color; Chemical composition; Weight; Pororoca.

\section{Resumen}

Dialium Guianense, conocido popularmente como Pororoca o Jutaí, es una fruta exótica cultivada en zonas de la región amazónica. Este trabajo tuvo como objetivo realizar la caracterización biométrica y fisicoquímica de frutos de Dialium Guyanense. Para la evaluación de los parámetros biométricos, se pesaron y midieron veinte frutos enteros 
(largo y ancho) individualmente. El análisis de color se realizó usando un colorímetro Delta. La composición aproximada y el valor calórico de la pulpa de la fruta se determinaron analizando humedad, cenizas, lípidos, proteína cruda, fibra cruda y carbohidratos. El resultado de los parámetros biométricos y del análisis de color mostró que la pororoca es un fruto pequeño $(21,09$ x $12,18 \mathrm{~mm}$ y $0,82 \mathrm{~g})$ con piel marrón y pulpa de color naranja. En cuanto a los resultados de la composición química, la pulpa de pororoca presentó $28.26 \%$ de humedad; $71,74 \%$ de materia seca; $3,37 \%$ de ceniza; $0,45 \%$ de lípidos; $2,77 \%$ de proteína cruda; $5,07 \%$ de fibra cruda; y $60,07 \%$ de carbohidratos. La estimación del valor calórico reveló que la fruta tiene $255 \mathrm{kcal}$ g-1. Se concluye que la caracterización fisicoquímica cobra importancia para brindar datos que puedan ser utilizados por las industrias para obtener nuevos productos alimenticios.

Palabras clave: Análisis biométrico; Color; Composición química; Peso; Pororoca.

\section{Introdução}

Dialium Guianense, popularmente conhecida como Pororoca, Jutaí, Guapagui, entre outras, é uma fruta exótica cultivada em áreas da região amazônica, podendo ser encontrada também em países como o México e Peru (Herrero-Jáuregui et al., 2009; Loureiro, 1971; Segura et al., 2018; Vargas-Simón et al., 2003; Vargas-Simón et al., 2018). Ela se caracteriza por ser uma fruta globosa, semelhante ao Tamarindo, e possui diâmetro de aproximadamente 1 a $2,2 \mathrm{~cm}$ de comprimento. Costuma apresentar cor acastanhada quando madura, pericarpo frágil e um sabor agridoce. Possui uma ou duas sementes com tamanhos que podem variar de 5 a 9 mm de comprimento (Vargas-Simón et al., 2003). Sua polpa é usada na alimentação para a fabricação de diversos produtos, como sucos, sorvetes, doces, etc. (Herrero-Jáuregui et al., 2009; Vargas-Simón et al., 2018).

Muito se discute sobre qual é a definição de frutas exóticas, Watanabe \& Oliveira (2014) "consideram como sendo frutas exóticas todas aquelas que apresentam o sabor diferenciado ou características diferenciadas que as distinguem dos demais no formato, cor e arquitetura da planta". As frutas exóticas desempenham um papel importante para o país, pois é a partir dela que o mercado vem crescendo na busca de novos sabores e produtos, além de trazer renda para os pequenos produtores (Rodrigues, 2009). A inserção de tais frutas na alimentação contribui para uma dieta saudável e balanceada, necessária para o aumento do valor nutricional na refeição.

Entende-se alimentação saudável como sendo aquela baseada em práticas alimentares que trazem benefícios para o ser humano e diminuem a possibilidade de que se apresentem doenças crônicas não transmissíveis. A Organização Mundial da Saúde (OMS) define que, para que seja considerada uma alimentação saudável, se consuma 400 g de frutas e hortaliças diariamente (Jaime et al., 2016). Pensando nisso, as frutas exóticas se apresentam como ótima opção, pois são novas no mercado e se diferenciam daquelas que já existem tanto em sabor, quanto em cor ou aroma.

Porém, apesar de seus altos valores nutritivos, ainda são escassos os trabalhos acerca de muitas frutas exóticas, o que faz com que haja um desperdício de potencial das plantas que ainda não são conhecidas, como a pororoca. A caracterização dessas frutas pode trazer para o país novas fontes de medicamentos, matérias-primas para uso industrial, melhoria de alimentos com pouco valor nutricional e uma maior variedade de aromas e sabores no comércio de frutas frescas (Negri et al., 2016).

Dados sobre a composição química de frutas são importantes para que a população conheça e possa consumir os nutrientes necessários para configurar uma alimentação saudável, para verificar a adequação nutricional na dieta da população no geral, para avaliar o valor nutricional de cada fruta, para que se desenvolvam pesquisas sobre as relações entre dietas e doenças e também, na indústria de alimentos (Gondim et al., 2005).

Segundo o Ministério da Saúde (Brasil, 2014, p.20), “conhecimentos gerados por estudos experimentais ou clínicos são importantes para a formulação de recomendações sobre alimentação na medida em que fornecem a base para se entender como diferentes componentes dos alimentos interagem com a fisiologia e o metabolismo".

Estima-se que, de toda a produção de frutas no Brasil, apenas $0,8 \%$ são de frutas exóticas pouco exploradas economicamente. De 3.000 espécies de frutas comestíveis na zona tropical, 920 são nativas da América e destas apenas 12 são 
exploradas economicamente. No entanto, salienta-se que, ao conhecer tais frutas, não se fale apenas da capacidade de alimentar, mas também do potencial aplicação nas áreas de produção de combustíveis, agroindustrial, medicinal, entre outras (Rodrigues, 2009).

Desta forma, tornam-se necessárias pesquisas que elucidem a caracterização química, física e sensorial da pororoca. Diante do exposto, este trabalho teve como objetivo realizar a caracterização biométrica e físico-química de frutos de Dialium Guianense.

\section{Metodologia}

\section{Amostras}

Os frutos de pororoca foram colhidos em março de 2018 por produtores do estado do Pará e analisados no Campus Novo Paraíso do Instituto Federal de Roraima. Os frutos foram selecionados, lavados em água corrente e descascados manualmente, onde foi separada a polpa das cascas e sementes. As amostras foram armazenadas em embalagens de polipropileno a $-20^{\circ} \mathrm{C}$ até a realização das análises.

\section{Parâmetros biométricos}

Para a avaliação dos parâmetros biométricos, vinte frutos inteiros de pororoca foram pesados individualmente em balança analítica para obtenção do peso médio do fruto. Os frutos também foram medidos utilizando um paquímetro digital para obter os dados de comprimento e largura do fruto, conforme Ferrão et al. (2013).

\section{Análise da cor}

A análise da cor da polpa e cascas da pororoca foi realizada em colorímetro Delta, utilizando o sistema de cor CIELab, iluminante D65 e ângulo de $10^{\circ}$. O processo de análise ocorreu por meio de quatro leituras da amostra no equipamento, onde foram determinados os parâmetros de cor a*, b*, Croma, luminosidade e Ângulo Hue.

\section{Composição química}

A determinação da composição centesimal da polpa de pororoca foi realizada segundo os métodos descritos na AOAC (2005) para a determinação de umidade, cinzas, lipídeos, proteína bruta, fibra bruta e carboidratos.

Para determinar o teor da umidade da fruta, utilizou-se o método de secagem em estufa com uso de temperatura de $105{ }^{\circ} \mathrm{C}$ até peso constante. Os valores de cinzas totais foram obtidos por incineração da amostra em mufla à $550{ }^{\circ} \mathrm{C}$ por 5 horas. O teor de lipídios foi obtido por meio de adaptação do método de extração a frio descrito por Bligh e Dyer (1959). A análise de proteína bruta foi realizada pelo método de Micro-Kjeldahl. A concentração de fibra bruta foi avaliada pela digestão em solução de ácido sulfúrico seguida de uma digestão com solução de hidróxido de sódio em digestor de fibra (MA444/CI). Os valores de carboidratos foram determinados pela diferença entre 100\% (massa total) e a soma das demais frações. As análises foram realizadas em triplicata.

\section{Valor calórico}

A estimativa do valor calórico foi calculada pela soma dos resultados referente a lipídios, carboidratos e proteínas multiplicados por seus fatores gerais de conversão (9, 4 e 4 kcal g-1, respectivamente).

\section{Análise estatística}

Os dados foram analisados por meio do cálculo de média e desvio padrão utilizando Microsoft Excel 2010. 


\section{Resultados e Discussão}

\section{Parâmetros biométricos}

Os resultados da avaliação biométrica de 20 frutos de pororoca estão apresentados na Tabela 1. Os valores apresentados na Tabela 1 corroboram com os dados observados por Pires et al. (2018), sendo que os referidos autores relataram frutos de pororoca mais leves $(0,79 \mathrm{~g})$, porém com valores médios de comprimento $(21,48 \mathrm{~mm})$ e largura (14,13 mm) ligeiramente superiores aos encontrados neste trabalho. Com estas medidas, a fruta caracteriza-se por possuir um tamanho pequeno, como pode ser observado na Figura 1.

Tabela 1 - Análise dos parâmetros biométricos da Pororoca (Dialium Guianense).

\begin{tabular}{lllll}
\hline & Média $^{\mathbf{1}}$ & Desvio padrão & Mínimo & Máximo \\
\hline Comprimento $(\mathrm{mm})$ & 21,09 & 2,06 & 16,61 & 26,07 \\
Largura (mm) & 12,18 & 0,85 & 10,64 & 13,86 \\
Peso $(\mathrm{g})$ & 0,8209 & 0,16 & 0,5430 & 1,0982 \\
\hline
\end{tabular}

${ }^{1}$ Média da análise de 20 frutos inteiros. Fonte: Autores (2021).

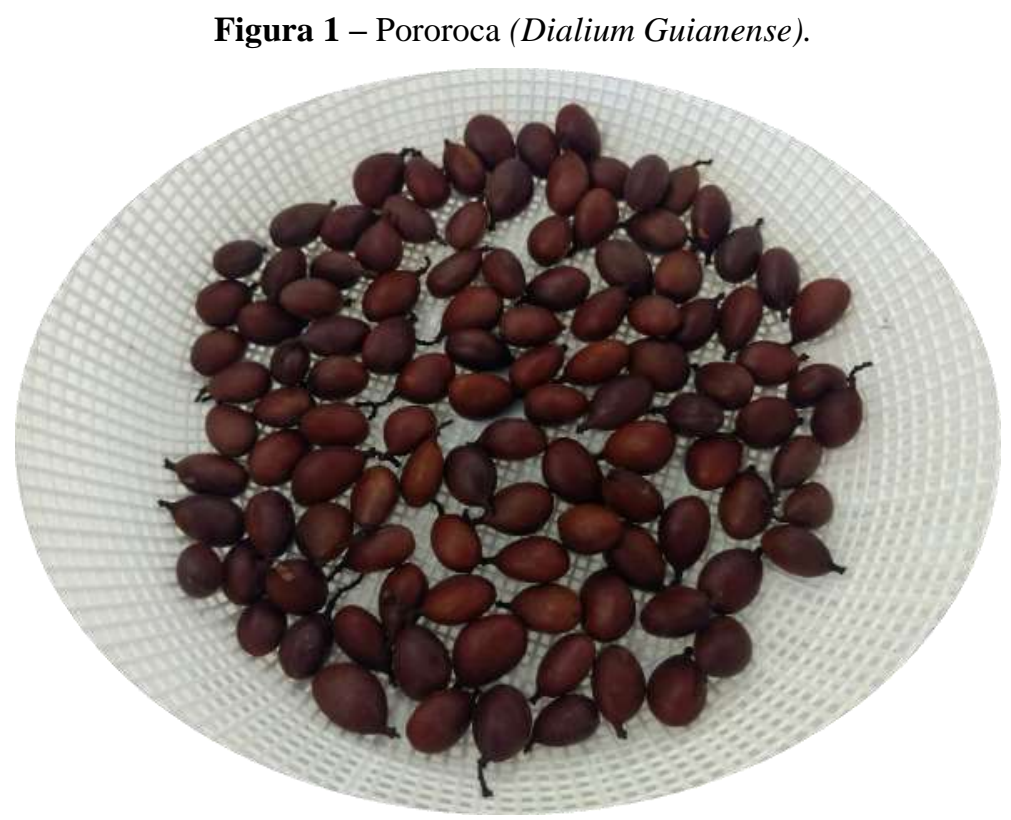

Fonte: Autores (2021).

As características biométricas da fruta estão de acordo com os valores relatados por Vargas-Simón et al., (2003), os quais descrevem os frutos como vagens de 1 a 2,2 cm de comprimento, globosas ou ovóides, indeiscentes, com pericarpo frágil, mesocarpo púbere acastanhado e sabor agridoce. Já Pires et al. (2018, p.258) apresentam a seguinte descrição para o fruto de Dialium Guianense: “fruto é um legume bacóide, indeiscente, obovado, marrom escura, pubescente; epicarpo cartáceo, mesocarpo delgado e papiráceo; endocarpo espesso de aspecto esponjoso e consistência carnosa, recobrindo toda a semente".

\section{Análise de cor}

Os valores dos parâmetros de cor analisados das amostras de polpa e casca da pororoca estão demonstrados na Tabela 2. 
Tabela 2 - Análise de cor da polpa e casca da pororoca (Dialium Guianense).

\begin{tabular}{lllll}
\hline Parâmetro & Polpa & Desvio Padrão & Casca & Desvio padrão \\
\hline Luminosidade & $40,83^{1}$ & 0,97 & 41,09 & 0,78 \\
$\mathrm{a}^{*}$ & 17,83 & 0,30 & 11,13 & 0,39 \\
$\mathrm{~b}^{*}$ & 23,86 & 2,37 & 22,43 & 0,74 \\
Croma & 33,51 & 2,16 & 25,05 & 0,83 \\
Ângulo Hue & 57,75 & 1,73 & 63,61 & 0,06 \\
\hline
\end{tabular}

${ }^{1}$ Média obtida de quatro análises. Fonte: Autores (2021).

Os resultados apresentados na tabela 2 demonstram que a fruta apresenta polpa com coloração alaranjada e casca de cor castanha amarronzada, característica evidente na Figura 2 e semelhante as informadas por Pires et al. (2018) que descrevem a cor dos frutos como marrom escuro, e por Vargas-Simón et al., (2003) os quais informam que o fruto possui mesocarpo púbere acastanhado.

Figura 2 - Cor da casca e polpa da pororoca (Dialium Guianense).

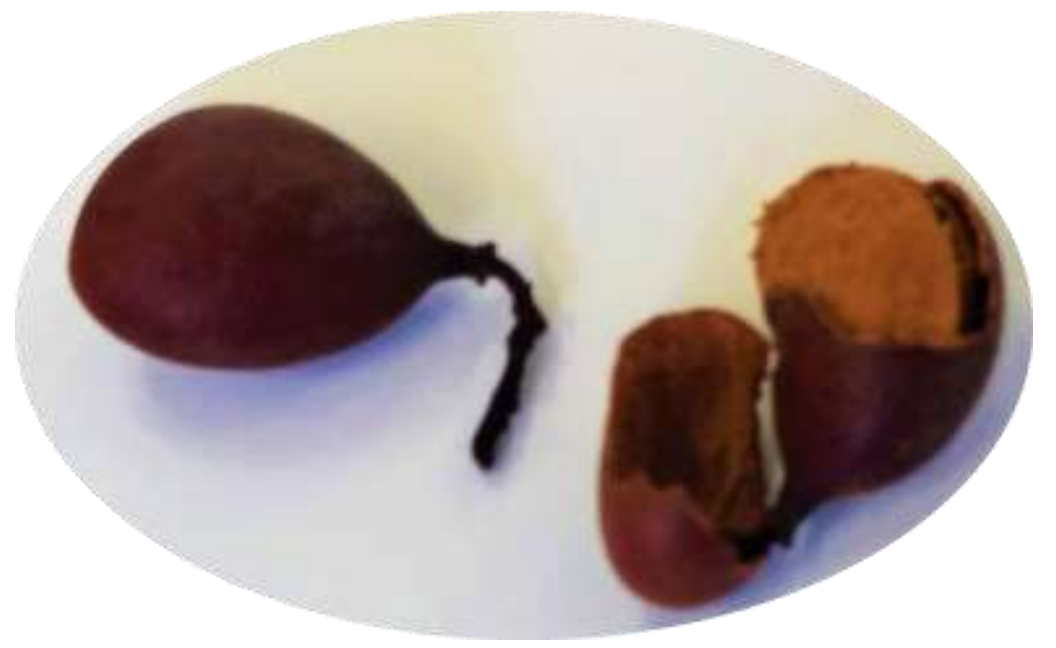

Fonte: Autores (2021).

\section{Composição química}

Na Tabela 3 é possível observar os dados da composição química da polpa da pororoca.

Tabela 3 - Composição química da polpa da pororoca.

\begin{tabular}{lll}
\hline Característica & Polpa da pororoca ${ }^{\mathbf{1}}$ & Desvio padrão $^{-}$ \\
\hline Umidade & $28,26 \%$ & - \\
Matéria seca & $71,74 \%$ & 0,30 \\
Cinzas & $3,37 \%$ & 0,07 \\
Lipídeos & $0,45 \%$ & 0,06 \\
Proteína bruta & $2,77 \%$ & 0,39 \\
Fibra bruta & $5,07 \%$ & 0,14 \\
Carboidratos & $60,07 \%$ & - \\
Valor calórico & $255 \mathrm{kcal} \mathrm{g}^{-1}$ & - \\
\hline
\end{tabular}

${ }^{1}$ Média obtida de três análises. Fonte: Autores (2021). 
Segundo Vargas-Simón et al., (2003) a pororoca se assemelha ao tamarindo. Quando comparados com os resultados de Taco (2011), observa-se que o tamarindo possui um teor de umidade de $22 \%$, menor do que o apresentado pela pororoca $(28,26 \%)$, porém as duas frutas apresentam umidade baixa quando comparadas com a maioria das frutas comercializadas, como maçã fuji (84\%), banana prata (72\%) e mamão formosa (86\%), condição favorável para a conservação dos frutos de pororoca. As frutas que possuem elevado teor de umidade são altamente perecíveis, pois a atividade de água está positivamente relacionada com o crescimento microbiano e com as reações bioquímicas de senescência do fruto. Dessa forma, frutas com alto teor de umidade requerem processos adicionais de conservação para prolongar sua vida útil, ocasionando maior gasto com métodos de conservação pós-colheita (Albuquerque et al., 2021, Ferrão et al., 2019).

Quanto ao teor de fibra $(5,07 \%)$, a polpa de pororoca apresentou superioridade nutricional quando comparada aos dados relatados pela TACO (2011) para frutas como maçã Fuji com casca (1,3\%), banana prata $(2,0 \%)$ e mamão formosa $(1,8 \%)$. As fibras proporcionam benefícios a saúde do organismo por auxiliarem no funcionamento do intestino, na redução do índice glicêmico, manutenção dos níveis de colesterol, entre outros (O’Shea et al., 2012).

O valor de lipídeos encontrado na polpa de pororoca $(0,45 \%)$, também é menor que o relatado para o tamarindo $(0,5 \%)$. Já a pororoca apresenta um maior valor de minerais totais $(3,37 \%)$ que os do tamarindo $(1,9 \%)$. Quanto à proteína e carboidratos o tamarindo apresenta maiores valores que os da pororoca $(3,20 \%$ e $72,5 \%$, respectivamente).

Dessa forma, os resultados indicam que, apesar de a pororoca possuir valores de composição centesimal distintos aos relatados no tamarindo, as frações nutricionais das duas frutas são semelhantes. Segundo Teixeira \& Novello (2020), a composição química das frutas depende de vários fatores como: genótipo, condições ambientais, práticas de cultivo, tratamento pós-colheita, condições de armazenamento; condições climáticas; condições de análise dos nutrientes (método de análise, preparo de amostra, equipamentos e reagentes); dentre outros. Por isso, há a necessidade de estudos que elucidem a composição físico-química de frutas ainda pouco conhecidas para impulsionar a comercialização e o setor frutífero.

Segundo Batista et al. (2020), o perfil alimentar da população adulta brasileira caracteriza-se pelo crescente e alto consumo de alimentos ultra processados e baixo consumo de alimentos ricos nutricionalmente, como as verduras e frutas, ocasionando problemas de saúde na população. Portanto, o fruto de pororoca apresenta-se como uma nova alternativa para favorecer a alimentação saudável e inclusão de novos sabores e atributos sensoriais para o uso na indústria alimentícia.

\section{Considerações Finais}

O presente trabalho demonstra que a pororoca pode ser inserida na dieta da população, uma vez que ela pode ser bem aceita por ser uma nova opção para aqueles que se interessam por frutas exóticas da região Amazônica. A caracterização físico-química se torna importante, pois, a partir dela, pode-se obter dados importantes que podem ser usados por indústrias de alimentos para a obtenção de novos alimentos ou insumos terapêuticos.

Dessa forma, são necessários estudos futuros sobre o processamento pós-colheita e a elucidação dos benefícios nutricionais da pororoca, assim como a determinação dos compostos bioativos, fitoquímicos e ácidos graxos.

\section{Agradecimentos}

Programa de Bolsas de Iniciação Científica do Instituto Federal de Educação, Ciência e Tecnologia de Roraima Pibict.

\section{Referências}

Albuquerque, A. P., Rodrigues, T. J. A., Cavalcante-Neto, J. L. \& Rocha, A. P. T. (2021). Utilização de polpa de frutas em pó carregadoras de probióticos como alimento funcional: aspectos gerais e perspectivas. Brazilian Journal of Food Technology. 24, e2019310. https://doi.org/10.1590/1981-6723.31019 
AOAC - Association Of Official Analytical Chemists. (2005). (17a ed.), Official Methods of Analysis. Arlington.

Bligh, E. G. \& Dyer, W. J. (1959). A rapid method of total lipid extraction and purification. Canadian Journal of Biochemistry and Physiology, $37,911-917$.

Batista, F. M., Sousa, A. P. S., Carvalho, L. M. F. \& Landim, L. A. S. R. (2020). Perfil nutricional e alimentar da população adulta brasileira: uma revisão integrativa. Research, Society and Development, 9 (11), e319119460. http://dx.doi.org/10.33448/rsd-v9i111.9460.

Brasil. Ministério da Saúde. Secretaria de Atenção à Saúde. Departamento de Atenção Básica. (2014). Guia alimentar para a população brasileira. (2a ed.), Brasília: Ministério da Saúde. https://bvsms.saude.gov.br/bvs/publicacoes/guia_alimentar_populacao_brasileira_2ed.pdf.

Ferrão, T. S., Ferreira, D. F., Flores, D. W., Bernardi, G., Link, D., Barin, J. S. \& Wagner, R. (2013). Evaluation of composition and quality parameters of jelly palm (Butia odorata) fruits from different regions of Southern Brazil. Food Research International, 54, 57-62. https://doi.org/10.1016/j.foodres.2013.06.002

Ferrão, T. S., Silva, I. P., Donadel, J. J. \& Wagner, R. (2019). Tendências no uso das micro-ondas: uma revisão crítica sobre a secagem de frutas e vegetais. In: Polese, V, Ferrão, T. S. \& Barros, R. P. Tópicos em Ciências Agrárias do IFRR. Kiron, 247-265. https://reitoria.ifrr.edu.br/pro-reitorias/pesquisa-e-posgraduacao/coordenacao-de-publicacao/e-books-1/topicos-em-ciencias-agrarias-do-ifrr/ciencias_agrarias_v1

Gondim, J. A. M., Moura, M. F. V., Dantas, A. S., Medeiros, R. L. S. \& Santos, K. M. (2005). Composição centesimal e de minerais em cascas de frutas. Ciência e Tecnologia de Alimentos, 25(4), 825-827.

Herrero-Jáuregui, C., García-Fernández, C., Sist, P.L., \& Casado, M.A. (2009). Conflict of use for multi-purpose tree species in the state of Pará, eastern Amazonia, Brazil. Biodiversity and Conservation, 18, 1019-1044. https://doi.org/10.1007/ s10531-008-9456-7

Jaime, P. C., Figueiredo, I. C., Moura, E. C. \& Malta, D. C. (2009). Fatores associados ao consumo de frutas e hortaliças no Brasil. Revista de Saúde Pública, $43(2), 57-64$

Loureiro, A. A. (1971). Contribuição ao estudo anatômico da espécie Dialium guianense (Aubl.) Sandw. (Leguminosae). Acta Amazônica, 1 (3), 85-87. https://doi.org/10.1590/1809-43921971013085.

Negri, T. C., Berni, P. R. A. \& Brazaca, S. G. C. (2016). Valor nutricional de frutas nativas e exóticas do Brasil. Biosaúde, 18 (2), 82-96. http://www.uel.br/revistas/uel/index.php/biosaude/article/view/27615

O'Shea, N., Arendt, E. K. \& Gallagher, E. (2012). Dietary fibre and phytochemical characteristics of fruit and vegetable by-products and their recent applications as novel ingredients in food products. Innovative Food Science and Emerging Technologies, 16, 1-10.

Pires, J. S., Mendes, A. M. S., Arruda, Y. M. B. C. \& Araujo, A. F. L. (2018). Aspecto morfológico do fruto, semente e desenvolvimento da plântula de Dialium guianense (aubl.) sandwith - Fabaceae, espécie utilizada na arborização urbana. IN: Anais do XXII Congresso Brasileiro de Arborização Urbana. 257261. https://www.sigrh.ufs.br/sigaa/verProducao?idProducao=1798719\&key=e501d6364ca8ec67e7b849c5e6773a2c

Rodrigues, L. A. (2009). Contribuição ao estudo bioquímico de frutas tropicais e exóticas produzidas no Brasil: pectina, açúcar e proteína. 2009.110 f. Dissertação (mestrado) - Universidade Estadual Paulista, Instituto de Química. http://hdl.handle.net/11449/88015

Segura, S., Fresnedo, J., Mathuriau, C., López, J., Andrés, J. \& Muratalla, A. (2018). The edible fruit species in Mexico. Genetic Resources and Crop Evolution. 65, 1767-1793.

Tabela Brasileira de Composição de Alimentos - TACO/ NEPA. (2011). NEPA- UNICAMP. https://www.cfn.org.br/wpcontent/uploads/2017/03/taco_4_edicao_ampliada_e_revisada.pdf

Teixeira, F. \& Novello, D. (2020). Aspectos físico-químicos, nutricionais e sensoriais da adição de subprodutos de frutos do gênero Citrus em produtos de gelificação: uma revisão sistemática. Research, Society and Development, 9 (3), e180932669. http://dx.doi.org/10.33448/rsd-v9i3.2669

Vargas-Simón, G., Pire, R., \& Lázaro-Dzul, M. (2018). Crecimiento plantular en condiciones de invernadero de la especie forestal Dialium guianense (Aubl.) Sandwith. Colombia Forestal, 21(1), 58-68. http://doi.org/10.14483/2256201X.11491

Vargas-Simón, G., Molina-Martínez, R. F., \& Moguel-Ordoñez, E. J. (2003). Caracterización del fruto, semilla y estudios de germinación del guapaque (Dialium guianense (Aubl) Sandwith). Universidad y Ciencia, 38(19), 77-83.

Watanabe, H. S. \& Oliveira, S. L. (2014). Comercialização de frutas exóticas. Revista Brasileira de Fruticultura, 36 (1), 23-38. https://doi.org/10.1590/0100$2945443 / 13$ 